\title{
Introduction to the Social-Technical Issues in Organizational Information Technologies Minitrack
}

\author{
Dragos Vieru \\ University of Québec \\ dragos.vieru@teluq.ca
}

\author{
Ulrika H. Westergren \\ Umeå University \\ ulrika.westergren@umu.se
}

\author{
Michael Knight \\ Texas A \& M - Kingsville \\ $\underline{\text { michael.knight } @ \text { tamuk.edu }}$
}

The Social-Technical Issues in Organizational Information Technologies minitrack focuses on information systems research areas impacting the intersection of humans and technology in an organizational context. Social issues related to organizational information technologies (IT) represents one of the most often discussed underpinnings in information systems research throughout the tenure of the IS field, and covers research topics that are the most aligned with the human factor in terms of information systems planning, development, innovation, implementation, and utilization.

Papers in the last several years have addressed issues such as distrust in information systems, technical security measures, diversity in organizational social media adoption, information security policies, as well as the inter-organizational dynamics of platform ecosystems and the establishing of IT-governance infrastructures in large healthcare corporations.

This year's minitrack focuses on three overarching themes: 1) Organizational culture and leadership; 2) Theoretical underpinnings and innovation practices; and 3) Management strategies. For HICSS-52, 12 papers were accepted for presentation.

The first four papers all explore different issues related to organizational culture and leadership. Paper 1: The social side of shadow IT and its impacts: investigating the relationship with social influence and social presence, shows how the use of shadow IT can positively influence communication and collaboration in the workplace. Paper 2: Facilitating Organizational Adoption of Sensor-Based Systems: Espoused Beliefs, Shared Assumptions and Perceived Values, examines the adoption of sensor-based systems through the lens of organizational culture theory. Paper 3: The Effect of CIO Virtues on $\mathrm{CIO}$ Role Effectiveness, explores the role of virtue ethics in effective and ethical CIO leadership. Finally, Paper 4: Out of Sight, Out of Mind: Turnover Intentions through an eLeadership, points to the importance of transformational leadership abilities in organizations that are either required to or choose to adopt information technologies.

The second set of papers focuses on the theoretical underpinnings and innovation practices present within the research domain. Paper 5: Understanding Virtual Embodiment: A Phenomenological Lens, contends that virtual embodiment is a valuable perspective for addressing the research contradictions in the area of virtual interactions. Paper 6: Change Agents and Resilient Practices: The Power of Symbolic Capital in a Post-Merger Integration Context, uses a practice perspective and the notion of symbolic capital to shed light on postmerger practices and their outcomes. Paper 7: The Role of Institutional Work in Platform Establishment: An Investigation of Digital Innovation Practices for Creating, Maintaining and Disrupting Institutions, takes a look at digital innovation practices and argues that one source of digital platform emergence is the recombinatorial innovation processes that individuals enact in organizational work practices. Paper 8: IT Infrastructures Maintenance and Evolution in Public Sector Organizations: Main Challenges, examines the public sector perspective on main organizational challenges related to the evolution and maintenance of IT infrastructures, as a first step in identifying critical issues to address within this research area.

The third and final set of papers delves into various management strategies related to organizational IT. Paper 9: Self-Organizing is not Self-Managing: A Case Study about Governance Challenges in an Agile IT Unit and its Scrum Projects, addresses the meaning of self-organizing versus self-managing practices, and their associated challenges on organizational governance processes. Paper 10: The Creativity Model of Age and Innovation with IT: How to Counteract the Effects of Age Stereotyping on User Innovation, proposes that the indirect effect of age through creative IT selfefficacy on user innovation can be lessened by contextual factors, namely job control, IT support, and user enjoyment. Paper 11: Prioritizing Tasks in 
Code Repair: A Psychological Exploration of Computer Code, takes a fresh look at task prioritization and its relation to efficiency and increased performance. Paper 12: Simulating the Impact of Cooperation and Management Strategies on Stress and Economic Performance, shows how management evaluation strategies that are aimed at achieving a balance between rewarding the cooperative behavior of the employees and their direct economic performance, influences stress levels, organizational profit, and other outcomes.

In sum, these 12 papers address current and relevant social-technical issues within organizational IT. In so doing they add to the knowledge base of the field and expand our understanding of the complex interplay of social and technical issues and how they affect organizational life. 